\title{
Stellar X-ray heating of planet atmospheres
}

\author{
C. Cecchi-Pestellini ${ }^{1}$, A. Ciaravella ${ }^{2}$, and G. Micela ${ }^{2}$ \\ 1 INAF - Osservatorio Astronomico di Cagliari, Strada 54, Loc. Poggio dei Pini, 09012 Capoterra (CA), Italy \\ e-mail: ccp@ca.astro.it \\ 2 INAF - Osservatorio Astronomico di Palermo, Piazza Parlamento 1, 90134 Palermo, Italy \\ e-mail: ciarave@astropa.unipa.it, giusi@astropa.unipa.it
}

Received 24 July 2006 / Accepted 28 August 2006

\section{ABSTRACT}

\begin{abstract}
Aims. To investigate the effects of the stellar X-ray irradiation on planet atmospheres, we study the X-ray transfer and energy deposition in a hydrogen rich gas.

Methods. We construct an accurate X-ray transfer model taking both photoionization and Compton scattering into account; the electron energy deposition is followed by tracking the discrete exchange processes between electrons and the gas mixture.

Results. Exospheric heating rates are derived as functions of the pressure in model atmospheres using a wide range of X-ray luminosity, spectral hardness representative of different stellar ages, and distances from the parent star. The computed heating rates suggest that X-ray irradiation might be an important heating source in planetary exospheres even at large distances from the parent star.
\end{abstract}

Key words. planetary systems - X-rays: stars - radiative transfer

\section{Introduction}

The early solar system experienced a much more active, hard, and bright X-ray emission from the Sun. Young solar type stars emit X-rays at a level three to four orders of magnitude higher than the present-day Sun, both during the pre-main sequence phase when the emission is dominated by intense daily or weekly flares (Feigelson et al. 2003; Favata et al. 2005), and during the first phases of the main sequence when a solar-like star has an X-ray luminosity $L_{\mathrm{X}}^{\star} \sim 10^{29}-10^{30} \mathrm{ergs} \mathrm{s}^{-1}$ (Micela 2002). Such an intense X-ray emission, as well as its rapid changing with age, could have strong effects on the formation and evolution of planetary systems. In particular. heating by energetic X-ray photons could affect the history of the composition and structure of planet atmospheres. High energy X-rays penetrate much deeper into gas clouds than do ultraviolet (UV) photons and are preferentially absorbed by heavy elements producing multiplycharged ions. The fast electrons produced by absorption of X-ray photons drive the chemistry and the heating of the gas.

The recent observations of an extended and hydrogenevaporating atmosphere for HD 209458b (Vidal-Madjar et al. 2003) has raised the question of the atmospheric stability against thermal escape at very short orbital distances (Lammer et al. 2003), and has renewed interest in the study of propagation of stellar radiation through planetary atmospheres. Since the work of Lammer et al. (2003), a few other models have been put forward (Yelle 2004; Baraffe et al. 2005; Tian et al. 2005) describing the effect of evaporation on the evolution of giant and terrestrial-like planets. Lammer et al. (2003) used the heatbalance equation to estimate an average exospheric temperature, while Yelle (2004) assumed that the extra energy acquired by a photoelectron in an ionization event is transferred to the ambient atmosphere with an efficiency of 63\% (Waite et al. 1983). All these studies include radiative transfer of stellar EUV (e.g.
Woods et al. 1998), but a detailed treatment of X-ray propagation and energy deposition is missing.

In this work we investigate the effects of X-ray energy deposition in the upper layers of planetary atmospheres of solar composition using a detailed X-ray transfer model and an accurate description of photoelectron energy deposition in order to derive heating rates across the planet exosphere. We assume a wide range of X-ray stellar luminosity so as to simulate the evolution of stellar emission with age. We construct an X-ray transfer model similar in many respects to those developed in the study of interstellar X-ray dominated regions (Maloney et al. 1996; Yan \& Dalgarno 1997). The investigation incorporates a thorough analysis of electron energy degradation in a gas mixture of $\mathrm{H}$, $\mathrm{H}_{2}$, and $\mathrm{He}$ of varying fractional abundances and electron content. A Monte Carlo code and an empirical gas photo-absorption cross-section has been developed by Smith et al. (2004) to estimate the order of magnitude for the fraction of energy that reaches the ground in terrestrial-like exoplanets. We describe the model in Sect. 2, present the results in Sect. 3, and discuss the implications in Sect. 4.

\section{X-ray transfer and energy deposition}

We consider a plane-parallel geometry in which a stratified model atmosphere is single-side illuminated by a normally incident X-ray flux and described by an exponential decrease in density with altitude $z$. This is an excellent approximation for the high energy photon transport, in which the energies considered are much higher than electronic binding energies, and radiative transfer is basically independent of the chemical and thermal profiles. At each grid point, the gas density is obtained by the scale height $H$ and by the assumed total column density in $\mathrm{g} \mathrm{cm}^{-2}$, which determines the number density of the particles $n_{0}$ at the ground level. 
In the radiative transfer calculation we consider photoelectric cross-sections of atomic hydrogen (Sobel'man 1991), helium (Yan et al. 1998), ionized helium (Osterbrock 1989), and molecular hydrogen (Yan et al. 1998). For heavy elements bound in molecules or molecular ions, the X-ray absorption cross-sections can be obtained approximately by adding the atomic crosssections. We adopt relativistic photoionization cross-sections of individual heavy elements and their ions given in Verner et al. (1993). The gas-effective X-ray photoabsorption cross-section $\sigma_{\mathrm{pa}}(E)$ is given by

$\sigma_{\mathrm{pa}}(E)=\sum_{i} f_{i} \sigma_{i}(E)$

where $f_{i}$ is the fractional concentration of the $i$ th species. Since, at high energies Compton ionization is more efficient than photoionization of $\mathrm{H}$ and $\mathrm{He}$ (Yan et al. 1998), we also include nonrelativistic Compton ionization cross-sections for $\mathrm{H}, \mathrm{H}_{2}$, and $\mathrm{He}$ (Jackson 1975). The primary ionization rate results

$\zeta_{1}=\int S_{\mathrm{X}}(E)\left[\sigma_{\mathrm{pa}}(E)+\sigma_{\mathrm{C}}\right] \mathrm{d} E$

where $S_{\mathrm{X}}$ is the local X-ray photon flux per unit energy, and $\sigma_{\mathrm{C}}$ the total Compton photoionization cross-section.

A unique feature of X-ray irradiation is that all the relevant processes are dominated by secondary ionizations generated by primary photoelectrons. This is a consequence of the large primary photoelectron energies. Actually, the secondary processes are far more important than the corresponding ionization, excitation, and dissociation events caused directly by X-rays. The total ionization rate $\zeta_{\mathrm{T}}$ is given by

$\zeta_{\mathrm{T}}=\zeta_{1}+\sum_{k} \int\left(\frac{\mathrm{d} \mathcal{N}_{1}(E)}{\mathrm{d} E} \times \mathcal{N}_{2}(\Delta E)\right)_{k} \mathrm{~d} E$

where the sum is over all ionization channels, $\mathrm{d} \mathcal{N}_{1}(E) / \mathrm{d} E$ is the primary electron differential spectrum, and $\mathcal{N}_{2}(\Delta E)$ the number of secondary ionizations produced as a fast primary particle of energy $\Delta E$ comes to rest. The energy degradation is characterized by the mean energy per ion pair $W$, which is the initial energy of the electron $\Delta E$ divided by $\mathcal{N}_{2}$. For high energies, $W$ is nearly constant, but it increases rapidly as the ionization threshold is approached (Dalgarno 1962). We follow the chain of discrete energy deposition events using the method described in Dalgarno \& Lejune (1971), Cravens et al. (1975), and Dalgarno et al. (1999). We adopt the electron impact cross-sections for excitation, ionization, dissociation, and elastic collisions listed in Dalgarno et al. (1999). Neglecting the primary ionization rate, the total ionization rate (per $\mathrm{H}$ nucleus) reads as

$\zeta_{\mathrm{T}} \approx f_{\mathrm{H}} \zeta_{\mathrm{H}}+f_{\mathrm{H}_{2}} \zeta_{\mathrm{H}_{2}}+f_{\mathrm{He}} \zeta_{\mathrm{He}}$

where

$\zeta_{m}=\int S_{\mathrm{X}}(E)\left[\sigma_{\mathrm{pa}}(E)\left(E-I_{m}\right)+\sigma_{\mathrm{C}}(E) \Delta E_{\mathrm{C}}\right] \frac{\mathrm{d} E}{W_{m}(E)}$

with $m \equiv \mathrm{H}, \mathrm{H}_{2}$, He. In Eq.(5) $I_{m}$ is the ionization threshold of the $m$ th species, and $\Delta E_{\mathrm{C}}$ the energy of released Compton electrons derived using a random walk technique (Rybicki \& Lightman 1979). The X-ray photoionization heating rate has the same form as Eq. (3) with $\mathcal{N}_{2}$ replaced by the fraction of primary photoelectron energy $\mathcal{E}_{Q}(\Delta E)$ going into gas heating

$Q_{X}=\sum_{k} \int\left(\frac{\mathrm{d} \mathcal{N}_{1}(E)}{\mathrm{d} E} \times \mathcal{E}_{Q}(\Delta E)\right)_{k} \mathrm{~d} E$.

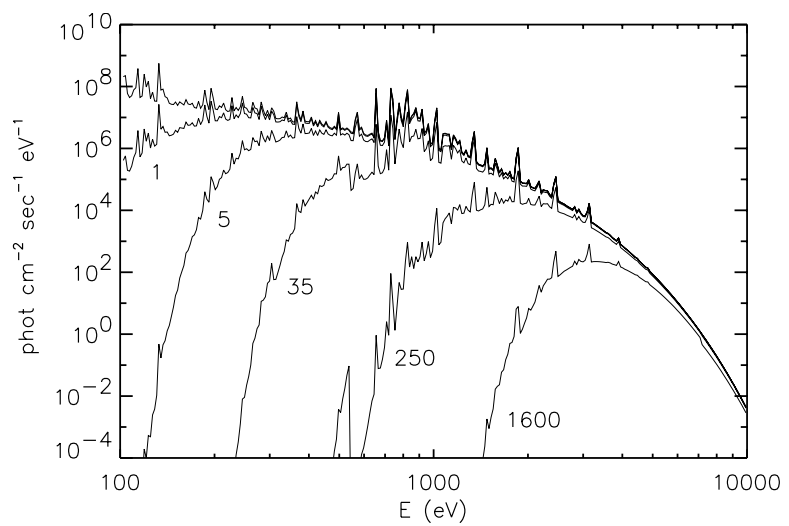

Fig. 1. Transmitted X-ray spectrum as a function of the energy at different of the atmospheric layers. The top curve is the incident stellar spectrum, obtained with a Raymond-Smith model at a temperature of $0.5 \mathrm{keV}$.

The physical and chemical state of a parcel of dense gas illuminated by a strong X-ray source should be scarcely sensitive to the actual shape of the X-ray spectrum, depending mostly on the $\mathrm{X}$-ray flux and gas density. However, to simulate spectra differing significantly in shape, we adopt Raymond-Smith models for the thermal emission of hot plasmas (Raymond \& Smith 1977). The local X-ray flux is

$S_{\mathrm{X}}(z)=S_{\mathrm{X}}^{\star} \exp \left[-\tau_{\mathrm{X}}(z)\right]$

where $S_{\mathrm{X}}^{\star}$ is the radiation field of the parent star reaching the upper boundary of the atmosphere, and $\tau_{X}$ the gas opacity

$\tau_{\mathrm{X}}(z)=\left(\sigma_{\mathrm{pa}}+\sigma_{\mathrm{C}}\right) \times\left[N_{\mathrm{H}}(\Delta z)-N_{\mathrm{H}}(z)\right]$,

$\Delta z$ being the atmosphere thickness. The absorption of the stellar $\mathrm{X}$-ray photons as they penetrate a sample atmosphere is shown in Fig. 1.

As the depth inside the atmosphere increases, the X-ray spectrum becomes progressively weaker and harder. The fast electrons produced in the process contribute to ionization, dissociation, excitation, and heating of the atmospheric gas.

\section{Results}

In this work we consider a solar-like parent star, where $M=M_{\odot}$ and $R=R_{\odot}$, and $\mathrm{X}$-ray luminosities $L_{\mathrm{X}}^{\star}$ ranging from $10^{29}$ to $10^{31} \mathrm{erg} \mathrm{s}^{-1}$ whose spectra are obtained with Raymond-Smith models at temperatures of $0.3,0.5$, and $1 \mathrm{keV}$, respectively. Thus, the brightest case corresponds to the hardest spectrum. The assumed range of X-ray luminosities and spectra accounts for the steep change with age of solar emission (Micela 2002). We modelled a Jupiter-like planet with surface gravity $g=$ $2.3 \times 10^{3} \mathrm{~cm} \mathrm{~s}^{-2}$, atmosphere width $\Delta z=1000 \mathrm{~km}$, pressure scale height $H=26 \mathrm{~km}$, and solar metallicities (Anders \& Grevesse 1989). In the calculations we assumed $f_{\mathrm{He}}=0.1$. We considered a minimal reaction network for $\mathrm{H}$ - and He-bearing species describing a chemistry initiated by X-ray-induced fast photoelectron ionization, Eq. (5), similar in many respects to that presented by Yelle (2004). Three representative values of the distance from the parent star, $D=0.05,5$, and $50 \mathrm{AU}$ were then selected.

Resulting heating rates through the planet atmosphere as functions of the pressure are shown in Fig. 2. Each panel corresponds to a distance from the parent star and contains nine 

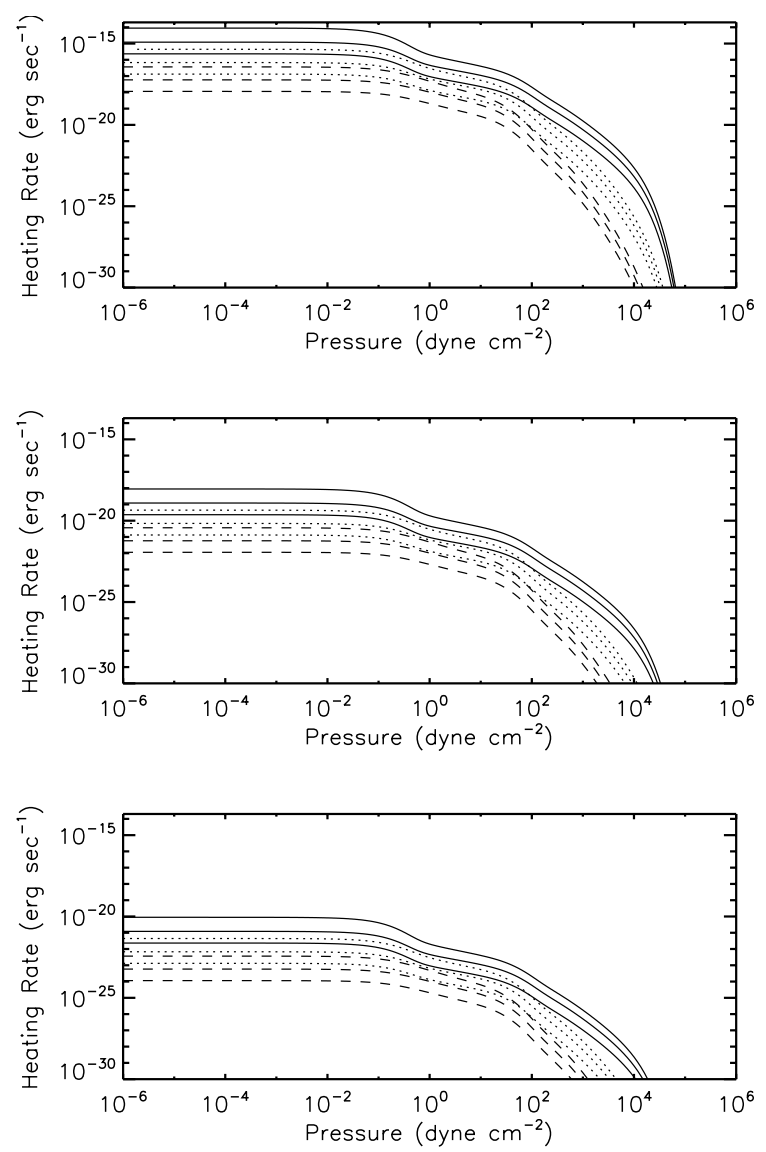

Fig. 2. X-ray heating of model atmospheres as functions of the pressure for a Jupiter-like planet located at $D=0.05 \mathrm{AU}$ (top panel), $D=5 \mathrm{AU}$ (middle panel), and $D=50 \mathrm{AU}$ (bottom panel). The X-ray luminosities of the parent star are $10^{29} \mathrm{erg} \mathrm{s}^{-1}$ (dashed lines), $10^{30} \mathrm{erg} \mathrm{s}^{-1}$ (dotted lines), and $10^{31} \mathrm{erg} \mathrm{s}^{-1}$ (solid lines). For each luminosity the electron fractions are $10^{-1}$ (upper curve), $10^{-3}$ (middle curve), and $10^{-6}$ (lower curve).

curves corresponding to three values of X-ray luminosity and three values of the gas electron content, $x_{\mathrm{e}}=10^{-1}, 10^{-3}$, and $10^{-6}$, which simulate a wide range of physical conditions from (almost) neutral to highly ionized gases. Although the hardness and intensity of the stellar spectrum determine the ionization degree of the planet atmosphere, we parametrized the electron content in order to highlight the effect of the electron concentration on the heating rate.

As expected the outer layers of the atmosphere experience the greatest heating, which is almost constant up to 0.1 dyne $\mathrm{cm}^{-2}$. Then the heating decreases significantly in the inner layers where only a negligible fraction of X-ray photons penetrate. For a model atmosphere the heating rate increases with the ionization of the gas, i.e. with electron fraction increasing from $x_{\mathrm{e}}=10^{-6}$ to $10^{-1}$. Figure 2 also shows that the heating rate scales linearly with the incident flux: a difference of three orders of magnitude in the distance from the parent star implies a difference of six orders of magnitude in the heating rate (e.g. compare the highest solid lines in the three panels). The heating rate decreases about linearly with the stellar X-ray luminosity. Thus the heating rate at the top of the atmosphere of a planet at $5 \mathrm{AU}$ changes from $10^{-18} \mathrm{erg} \mathrm{s}^{-1}$ to $10^{-20} \mathrm{erg} \mathrm{s}^{-1}$ when the $\mathrm{X}$-ray luminosity of the parent star changes from $10^{31} \mathrm{erg} \mathrm{s}^{-1}$ to $10^{29} \mathrm{erg} \mathrm{s}^{-1}$.
Table 1. Fitting parameters of Eq. (11).

\begin{tabular}{ccccccc}
\hline \hline$a$ & $b$ & $\alpha$ & $\sigma_{0}(\mathrm{Mb})$ & $\gamma$ & $E_{-}(\mathrm{keV})$ & $c \Gamma(\beta)$ \\
\hline 0.98 & 38 & 0.76 & 2.3 & 3.2 & 0.1 & 0.5 \\
\hline
\end{tabular}

In order to use our computed X-ray heating rates in atmospheric modeling, we derive an analytical expression starting from the approximate form

$Q_{\mathrm{X}} \approx \overline{\mathcal{E}_{Q}} \zeta_{1}$

where $\zeta_{1}$ is the local X-ray energy deposition rate, i.e. the primary ionization rate given in Eq. (2). We set

$\zeta_{1}=\frac{L_{\mathrm{X}}^{\star}}{4 \pi D^{2}} \times \int_{E_{-}}^{E_{+}} \sigma_{\mathrm{pa}} \exp \left(-\sigma_{\mathrm{pa}} \Delta N_{z}\right) \mathrm{d} E$

where $\Delta N_{z}=N_{\mathrm{H}}(\Delta z)-N_{\mathrm{H}}(z)$. The total photoelectric crosssection can be roughly described by the power-law fit $\sigma_{\mathrm{pa}}(E)=$ $\sigma_{0}(E / \mathrm{keV})^{-\gamma}$ (Maloney et al. 1996). Defining the effective optical depth $\tau_{0}(z)=\sigma_{0} \Delta N_{z}$ and adopting the fitting representation of the heating efficiency proposed by Dalgarno et al. (1999) $\overline{\mathcal{E}_{Q}}=1-a /\left(1+b x_{\mathrm{e}}^{\alpha}\right)$, valid for $x_{\mathrm{e}} \lesssim 0.1$, we obtain for $E_{+} \gg E_{-}$

$$
\begin{aligned}
Q_{\mathrm{X}}(\mathrm{ergs} / \mathrm{s}) & =3.5 \times 10^{-16}\left(1-\frac{a}{1+b x_{\mathrm{e}}^{\alpha}}\right) \frac{\left(\sigma_{0} / \mathrm{Mb}\right) \mathcal{L}_{\mathrm{X}}^{\star}}{(D / \mathrm{AU})^{2}} \\
& \times \begin{cases}\frac{1}{\gamma-1} E_{-}^{\gamma-1} & \tau_{0}(z) \rightarrow 0 \\
(\beta-1) \tau_{0}(z)^{-\beta} c \Gamma(\beta) & \tau_{0}(z) \geq \overline{\tau_{0}}\end{cases}
\end{aligned}
$$

where $\mathcal{L}_{\mathrm{X}}^{\star}$ is the X-ray stellar luminosity in units of $10^{30} \mathrm{ergs} / \mathrm{s}$, $\beta=1 / \gamma+1, c$ is a fitting constant, and $\Gamma$ is the Euler function (Abramovitz \& Stegun 1965). Here, $\overline{\tau_{0}}$ corresponds to the gas column density

$\overline{\Delta N_{z}}=\sigma_{0}^{-1}\left[\frac{(\beta-1)(\gamma-1) c \Gamma(\beta)}{E_{-}^{\gamma-1}}\right]^{1 / \beta}$.

Equation (11) is accurate within 50\% for column densities $\Delta N_{z} \lesssim 10^{21} \mathrm{~cm}^{-2}$. Fitting parameters are reported in Table 1 .

\section{Conclusions}

To estimate the X-ray contribution to exospheric heating in hydrogen-rich planetary atmospheres, we use a detailed description of the X-ray transfer and (photo) electron energy deposition in a gas of solar composition. We derive heating rates as functions of the altitude, for several assumptions on atmospheric boundary conditions.

As little detail has been given in previous works about the spectrum of the stellar radiation propagating through the planetary atmosphere, it is not easy to make a comparison between our heating rates and those present in the literature. Some authors refer explicitly to the solar spectrum presented by Woods et al. (1998) in which the energy deposition is by far dominated by the EUV and Ly $\alpha$, and thus X-rays are likely to be excluded. For the brightest stellar luminosities assumed in our calculations, the resulting heating rates (erg/s) are significantly higher than those presented by Yelle (2004) for planets at the same distance from the parent star $(D=0.05 \mathrm{AU})$. As a consequence, $\mathrm{X}$-rays might play an large role in the upper layers of planetary atmospheres. 


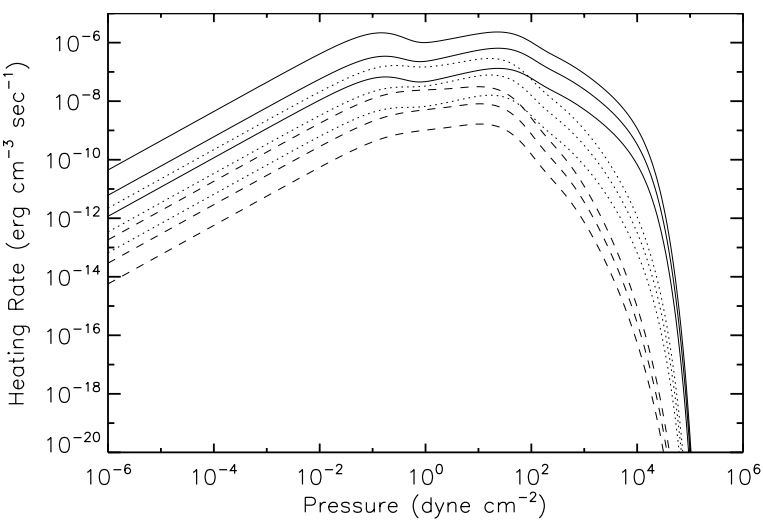

Fig. 3. X-ray heating of model atmospheres as functions of the pressure for a Jupiter-like planet located at $D=5 \mathrm{AU}$. The profiles in the this figure have been obtained as the product of the heating rates of Fig. 2 (middle panel) and the density profiles along the atmosphere.

Moreover, the high derived exospheric heating rates are not characteristic of only the close-in exoplanets but instead appear to pertain to all hydrogen-dominated planet atmospheres $(D \leq 50 \mathrm{AU})$, although the exospheric heating rate, as a general trend, is linearly dependent on the stellar X-ray flux and luminosity.

Though we focus on higher X-ray stellar luminosities than that of the present Sun, our results may have implications for the current physical conditions of the solar system (cf. Fig. 3). We will address this point in a forthcoming paper.

\section{References}

Abramowitz, M., \& Stegun, I. A. 1965, Handbook of mathematical functions (Dover: New York)

Anders, E., \& Grevesse, N. 1989, Geochim. Cosmochim. Acta, 53, 197

Baraffe, I., Chabrier, G., Barman, T. S., et al. 2005, A\&A, 436, L47

Cravens, T. E., Victor, G. A., \& Dalgarno, A. 1975, Planet. Space Sci., 23, 1059

Dalgarno, A. 1962, in Atomic and Molecular Processes, ed. D. R. Bates (Academic Press, New York)

Dalgarno, A., \& Lejeune, G. 1971, Planet. Space Sci, 19, 1653

Dalgarno, A., Yan, M., \& Liu, W. 1999, ApJS, 125, 237

Favata, F., Flaccomio, E., Reale, F., et al. 2005, ApJS, 160, 469

Feigelson, E. D., Gaffney, J. A. Garmire, G., Hillenbrand, L. A., \& Townsley, L. 2003, ApJ, 584, 911

Jackson, J. D. 1975, Classical Electrodynamics (New York: Wiley)

Lammer, H., Selsis, F., Ribas, I., et al. 2003, ApJ, 598, L121

Maloney, P. R., Hollenbach, D. J., \& Tielens, A. G. G. M. 1996, ApJ, 466, 561

Micela, G. 2002, in ASP Conf. Ser., 269, The evolving Sun and Its Influence on Planetary Environments, ed. B. Montesinos, A. Gimenez, \& E. F. Guinan (San Francisco: ASP), 107

Osterbrock, D. E. 1989, Astrophysics of Gaseous Nebulae and Active Galactic Nuclei (Sausalito, University Science Books)

Raymond, J. C., \& Smith, B. W. 1977, ApJS, 35, 419

Rybicki, G., \& Lightman, A. P. 1979, Radiative Processes in Astrophysics (New York: Wiley-Interscience)

Smith, D. S., Scalo, J., \& Wheeler, J. C. 2004, Icarus, 171, 229

Sobel'man, I. 1991, Atomic Spectra and Radiative Transitions (Berlin, Springer) Tian, F., Toon, O. B., Pavlov, A., \& De Sterck, H. 2005, ApJ, 621, 1049

Verner, D. A., Yakovlev, D. G., Band, I. M., \& Trzhaskovskaya, M. B. 1993, Atomic Data and Nuclear Data Tables, 55, 233

Vidal-Madjar, A., Lecavelier des Etangs, Désert, J.-M., et al. 2003, Nature, 422, 143

Waite, J. H., Cravens, T. E., Kozyra, J., et al. 1983, J. Geophys. Res., 88, 6143

Woods, T. N., Rothman, G. J., Bailey, S. M., \& Worden, J. R. 1998, Sol. Phys., 177,133

Yan, M., \& Dalgarno, A. 1997, ApJ, 481, 296

Yan, M., Sadeghpour, H. R., \& Dalgarno, A. 1998, ApJ, 496, 1044

Yelle, R. V. 2004, Icarus, 170, 167 